\title{
Blutbad hinterm Steuer
}

— In Vorarlberg kamen die Notfälle normalerweise mit der Rettung oder mittels Hubschrauber zu uns, insbesondere schwerere Verletzungen. Außer einmal: Ein ca. 40-jähriger Mann aus dem Bregenzer Wald (ca. 40 min von unserem Spital entfernt) stellt sich bei uns vor, über und über voll Blut. Er sei in die Kreissäge geraten, Bein und Arm habe es „etwas" erwischt. Drei Pfleger und ich waren voll beschäftigt, die Wunden zumin- dest zu tamponieren. Wie sei er denn hereingekommen? "Ich habe mich ins Auto gesetzt, bis die Rettung bei uns ist, das hat mir zu lange gedauert ..."

Ein Wunder, dass dieser Mann noch fahren konnte. Ich erinnere mich nicht mehr an alle Details genau, jedenfalls hatte der Mann ein Riesenglück und die Verarztung ging ohne größere Probleme vonstatten, ein kleinchirurgischer Eingriff und Wund- nähte konnten alle in der Ambulanz erfolgen. Allerdings haben wir ihn dann natürlich zur Beobachtung hierbehalten und ihn nicht mehr selbstständig heimfahren lassen.

Mich hätte aber noch interessiert, wie das Auto ausgeschaut hat nach dieser Blutbadfahrt - und wer es anschließend putzen durfte...

Dr. med. Claudia Petroni, Sarntal, SÜdtirol -

\section{Darf's denn auch was Christlich's sein?}

— Folgendes Gespräch belauschte ich auf dem Flur eines städtischen Krankenhauses im pietistischen deutschen Südwesten:

"Ich wusste gar nicht, dass Herr Schierle im Krankenhaus ist", sagte die Dame mit dem Dutt zur anderen. „Was hat er denn?" „Er hat's mit dem Bauch." „Ja so was. Den muss ich heute auch einmal besuchen. Da weiß man aber gar nicht, was man ihm mitbringen kann.“ "Jedenfalls nichts zum Essen und auch koi Fläschle Wein.. "Was kann ich ihm denn sonst mitbringen? Vielleicht ein Buch?" "Das wäre eine gute Idee. Lesen tut er gern", sagte die andere.
„Darf's denn auch was Christlich's sein?", witterte die Dame mit dem Dutt ihre missionarische Chance.

"Noi, noi, net nötig", bereitete die andere dem Missionseifer ein jähes Ende. „Es geht ihm scho wieder besser."

Dr. MED. RAINER HAKIMI, STUTTGART • 